\title{
ANS54: A Computer Subroutine for Predicting Fission Gas Release
}

W. N. Rausch

F. E. Panisko

August 1979

Prepared for the

Core Performance Branch

Nuclear Regulatory Commission

Pacific Northwest Laboratory

Operated for the U.S. Department of Energy

by Battelle Memorial Institute 


\title{
NOTICE
}

This repon was prepared as an account of work sponsored by the United States Government. Neither the United States nor the United States Nuciear Regulatory Commission, nor any of their employees, nor any of their contractors, subcontractors, or their employees, makes any warranty, express or implied, or assumes any legal liability or responsibility for the accuracy, completeness or usefulness of any information, apparatus, product or process disclosed, or represents that its use would not infringe privately owned rights.

\author{
PACIFIC NORTHWEST LABORATORY \\ operated by \\ BATTELLE \\ for the \\ UNITED STATES DEPARTMENT OF ENERCY \\ Under Contract EY-76-C-06-1830
}

\author{
Printed in the United Sutes of America \\ Available from \\ National Techniai information Service \\ Unined States Department of Commerce \\ 3255 Port Royal Road \\ Springtield, Virginia 2215 ?
}

Price: Primed Copy \$

$\because$ Microfiche $\$ 3.00$

NTIS

-pages

Selling Price

$001-025 \quad 34.00$

$025-050 \quad 3.50$

OST- $055 \quad \$ 5.25$

(76-100 $\quad 56.00$

$101-125$. 56.50

$125-150 \quad 5725$

$151-175 \quad 28.00$

$176-200 \quad 59.00$

$201-225 \quad 59.25$

$225-250 \quad 5950$

251.DS S10.J5

DE-300 $\quad$ \$17.00 
ANS54: A COMPUTER SUBROUTINE FOR PREDICTING FISSION GAS RELEASE

by

W. N. Rausch

F. E. Panisko

\author{
August 1979 \\ Prepared for \\ the Core Performance Branch \\ Division of Systems Safety \\ Office of Nuclear Reactor Regulation \\ U.S. Nuclear Regulatory Commission \\ under a related services agreement \\ with the Department of Energy \\ under Contract EY-76-C-06-1830
}

Pacific Northwest Laboratory

Richland, Washington 99352 



\section{PREFACE}

Pacific Northwest Laboratory(PNL) and Idaho National Engineering Laboratory are collaborating on the development of a new steady-state fuel performance computer code, FRAPCON-2. The Core Performance Branch of the U.S. Nuclear Regulatory Commission requested that the fission gas release model proposed by the Working Group ANS-5.4 of the American Nuclear Society be included in the code. PNL, operated for the Department of Energy by Battelle Memorial Institute, has programmed the new gas release model(ANS54) and it will be included in FRAPCON-2.

This report summarizes the model developed by the ANS committee. It also shows a comparison of model predictions with the light water reactor(LWR) low burnup data and liquid metal fast breeder reactor(LMFBR) high burnup data that were used in the model development. A listing of the ANS54 subroutine is included. 


\section{ACKNOWLEDGMENT}

We wish to thank the Core Performance Branch of the Nuclear Regulatory Commission, Division of Systems Safety, for their support of this work. Mr. J. C. Voglewede and Dr. R. 0. Meyer of that branch were helpful in supplying source material and in reviewing the draft. Mr. C. R. Hann of PNL also made instructive comments on the draft. 
ANS54: A COMPUTER SUBROUTINE FOR PREDICTING FISSION GAS RELEASE

(W. N. Rausch, F. E. Panisko)

During the irradiation of reactor fuel rods, gaseous fission products are produced in the fuel. A fraction of these fission gases is released to the gap, i.e., outside of the fuel pellets yet inside the fuel rod cladding. This report presents a FORTRAN subroutine (ANS54) which calculates the fractions of fission gas (both stable and radioactive isotopes) released by the fuel. The routine is based on the model described in a proposed ANS -5.4 fission product release standard. (a) The subroutine is the gas release model for FRAPCON-2, but may, of course, be used with any steady-state fuel performance code. $(b, c)$

\section{Model Summary}

The fractional fission gas release is calculated (on a local basis only), as a function of temperature, time, and burnup. The fuel is divided into radial and axial nodes according to the ANS standard. A user requirement is that the time step sizes be such that the burnup increments do not exceed 2000 Mid/MTM.

The routine is divided into two main sections, one to model release of the stable isotopes and the other to model release of the short-lived isotopes. There are high and low temperature models for both the stable and radioactive fission products. The code always calculates the release using both high and low temperature models and then uses the larger release.

I.

The stable fission gas (high temperature model) cumulative total release fraction for a fuel volume after step $K$ is defined as:

$(a)_{R}$. 0. Meyer, "Proposed ANS-5.4 Standard", ANS 5.4 Committee Correspondence, (August 30, 1979).

(b) F. E. Panisko, letter report to J. C. Voglewede (April 12, 1979).

(c) W. N. Rausch, letter report to J. C. Voglewede (May 25, 1979). 


$$
\left.F_{K}=1-\sum_{i=1}^{K-1}\left[B_{i}\left(\tau_{i} g_{i}-\tau_{i+1} g_{i+1}\right) / D_{i}^{\prime}\right]+B_{K} \Delta t_{K} g_{K}\right\} \sum_{i=1}^{K} B_{i} \Delta t_{i} .
$$

with

$$
\begin{array}{ll}
\tau_{i}=\sum_{j=i}^{K} D_{j}^{\prime} \Delta t_{j} & \\
g_{i}=g\left(\tau_{i}\right)=1-4 \sqrt{\tau_{i} / \pi}+3 \tau_{i} / 2 & \text { for } \tau_{i}<0.1 \\
g_{i}=g\left(\tau_{i}\right)=\frac{1}{15 \tau_{i}}-\frac{6}{\tau_{i}}\left(\sum_{n=1}^{3} \frac{\exp \left(-n^{2} \pi^{2} \tau_{i}\right)}{n^{4} \pi^{4}}\right) & \text { for } \tau_{i}>0.1 \\
D_{i}^{\prime}=\left[\left(D_{0} / a^{2}\right) \exp \left(-Q / R T_{j}\right] \times 100^{B u_{i} / 28000}\right. &
\end{array}
$$

Where

$$
\begin{aligned}
F_{K} & =10 c a l \text { cumulative fractional gas release through time step } K \\
B_{i} & =10 \mathrm{cal} \text { fission gas production rate during the } i \frac{\text { th }}{\text { time step }} \\
\Delta t_{j} & =1 \text { ength of } i \frac{\text { th }}{\text { time step (seconds) }} \\
Q & =72,300 \mathrm{cal} / \mathrm{mol} \\
R & =1.987 \mathrm{cal} / \mathrm{mol}-{ }^{\circ} \mathrm{K} \\
T_{i} & =\text { temperature }\left({ }^{\circ} \mathrm{K}\right) \text { during the } i \frac{\text { th }}{} \text { time step } \\
B u_{j} & =\text { accumulated local burnup (MWd/MTM) at the midpoint of the } i \frac{\text { th }}{\text { time step }} \\
D_{0} / a^{2} & =0.61 \mathrm{sec}^{-1} \\
K & =\text { current time step }
\end{aligned}
$$

Equation 1 is rewritten in the code as:

$$
F_{K}=1-\frac{\sum_{i=2}^{k}\left(\frac{B_{i}}{D_{i}^{T}}\left(\tau_{i} g_{i}-\tau_{i+1} g_{i+1}\right)\right)}{\sum_{i=2}^{k} \quad B_{i} \Delta t_{i}}
$$

with

$$
{ }^{\tau_{K+1}}=g_{K+1}=0
$$


The FORTRAN coding uses only a $\tau_{1}\left(g_{1}\right)$ and $\tau_{2}\left(g_{2}\right)$, but these are updated for eacn time step. $\tau_{1}$ always represents $\tau_{i}$ and $\tau_{2}$ aiways represents $\tau_{i+1}$. The summations begin at $i=2$, because the first time step is assumed to be very sma11. (Most fuel codes require a very short, low power time step as the first "warm-up" step. FRAPCON's is built into the code and does not appear as output.)

II.

The low temperature model for the stable fission gas release is dependent only on burnup. The fraction fission gas released is:

$$
F=1 \times 10^{-7} \mathrm{Bu}
$$

where

$$
\mathrm{Bu}=\text { rod average accumulated burnup (MWd/MTM) }
$$

III.

The radioactive fission gas release high temperature model assumes that power and temperature have remained constant for several half-lives. The fraction released is a function of time, temperature and burnup, and is also dependent on half-life. The release fraction for isotope $i$ is:

$$
\begin{aligned}
& F_{i}=\frac{3}{T-\exp \left(-\mu_{j} \tau_{i}\right)}\left[\frac{1}{\sqrt{\mu_{1}}}\left[\operatorname{erf}\left(\sqrt{\mu_{j} \tau_{j}}\right)-2 \sqrt{\mu_{j} \tau_{j} / \pi} \exp \left(-\mu_{j} \tau_{j}\right)\right]\right. \\
& \left.-\frac{i-\left(1+\mu_{j} \tau_{j}\right) \exp \left(-\mu_{i} \tau_{j}\right)}{\mu_{i}}\right] \quad \text { for } \tau_{j} \leq 0.1
\end{aligned}
$$

and

$$
\begin{array}{r}
F_{i}=3\left[\frac{1}{\sqrt{\mu_{i}}} \operatorname{coth}\left(\sqrt{\mu_{i}}\right)-\frac{1}{\mu_{i}}\right]-\frac{6 \mu_{i}}{\exp \left(\mu_{i} \tau_{i}\right)-1}\left(\sum_{n=1}^{3} \frac{1-\exp \left(-n^{2} \pi^{2} \tau_{j}\right)}{n^{2} \Pi^{2}\left(n^{2} \Pi^{2}+\mu_{i}\right)}\right) \\
\text { for } \tau_{i}>0.1
\end{array}
$$

with

$$
\begin{aligned}
& \mu_{j}=\lambda_{j} / D^{\prime} \\
& \tau_{i}=D^{\prime} t
\end{aligned}
$$


where

$$
\begin{aligned}
\lambda_{i} & =\text { decay constant for isotope } i\left(\mathrm{sec}^{-1}\right) \\
t & =\text { total accumulated irradiation time (seconds) } \\
D^{\prime} & =\left[\left(D_{0} / a^{2}\right) \exp (-Q / R T)\right] \times 100 \mathrm{Bu} / 2800 \\
\text { erf } & =\text { error function } \\
B u & =\text { total accumulated burnup (MWd/MTM) } \\
T & =\text { temperature }\left({ }^{\circ} \mathrm{K}\right) \text { constant for previous four half-lives }
\end{aligned}
$$

IV.

The low temperature radioactive fission gas release for isotope $i$ is defined by:

$$
F_{i}=\left(1 / \lambda_{i}\right)\left[1 \times 10^{-7} \sqrt{\lambda_{i}}+2 \times 10^{-12} \mathrm{P}\right]
$$

where

$$
\begin{aligned}
P & =\text { specific power (megawatts per metric ton heavy metal) } \\
\lambda_{i} & =\text { decay constart }\left(\mathrm{sec}^{-1}\right)
\end{aligned}
$$

The radioactive fission gas release fraction is not needed in predicting the steady state fuel rod behavior in FRAPCON-2; it is calculated for informational purposes only. The results are output as a table and a plot of fractional release versus half life for each time step. The output subroutine, GASPLT, can be called from the ANS54 subroutine or from the main program.

\section{Model Development}

The model was developed by the ANS-5.4 working group on fission gas release. $1,2,3$

\section{Comparison with Data}

Although FRAPCON-2 is not yet sufficiently developed to check the entire code against experimental results, the ANS-5.4 high temperature stable fission gas release model was compared to the data base from which the model was developed. This data base included all the rods used to develop the Beyer-Hann 
fission gas release model plus 19 Hanford Engineering Development Laboratory (HEDL) LMFBR pins irradiated in Experimental Breeder Reactor(EBR) II.

The predicted and reported values are given in Tables 1 and 2 for the Beyer-Hann(LWR) and HEDL(LMFBR) data ${ }^{2}$ respectively. These tables give the pin identification and reported power, burnuo, fuel surface and centerline temperatures, and gas release. The predicted fuel temperatures used by the ANS model were calculated utilizing reported power, fuel surface temperature, fuel diameter, and enrichment. The centerline temperatures calculated for use by the model appear as $\mathrm{CL}^{\star}$.

Figure 1 presents the fission gas release predictions plotted against the reported values for the Beyer-Hann data set. Figure 2 plots the same information for the EBR-II pins. The ANS -5.4 model generally overpredicts the releases of the LWR pins, but tends to underpredict the high (over 40,000 MWd/MTM) burnup LMFBR data.

A comparison with other data is necessary to properly evaluate the model, but the information presented here should help the user to evaluate the predictions made by the ANS-5.4 stable high temperature gas release model.

\section{Fission Gas Release Subcode ANS54 Listing}

A listing of the computer subcode ANS54 is presented in Table 3 . A listing of the output subroutine GASPLT is included in Table 4 ,

\section{References}

1. R. A. Lorenz, "ANS-5.4 Fission Gas Release Model III. Low Temperature Release", ANS Topical Meeting on Light Water Reactor Fuel Performance, (April 1979) pp 336-345

2. L. D. Noble, "ANS-5.4 Fission Gas Model I. Noble Gases at High Temperature", ANS Topical Meeting on Light Water Reactor Fuel Performance, (April 1979) pp $321-327$

3. "Status Report: ANS5.4 Fuel Plenum Gas Activity (N218) (Fission Product Release From UO $\mathrm{F}_{2}$ Fuel)", (April 1977), (Available from S. E. Turner, Southern Science Applications, P.0. Box 10, Dunedin, Florida 33528) 


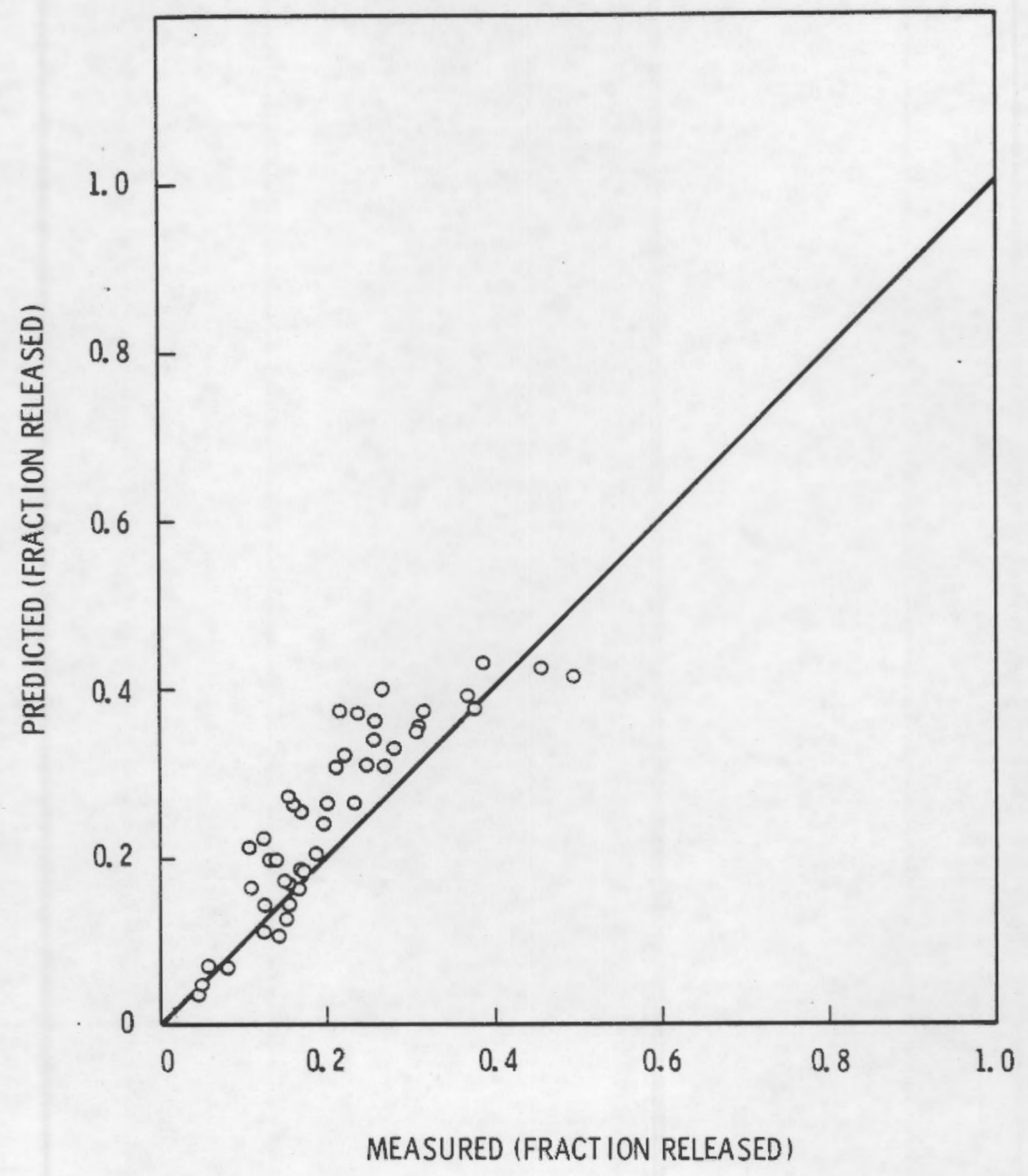

FIGURE 1. Comparison of ANS-5.4 With Low Burnup Data 


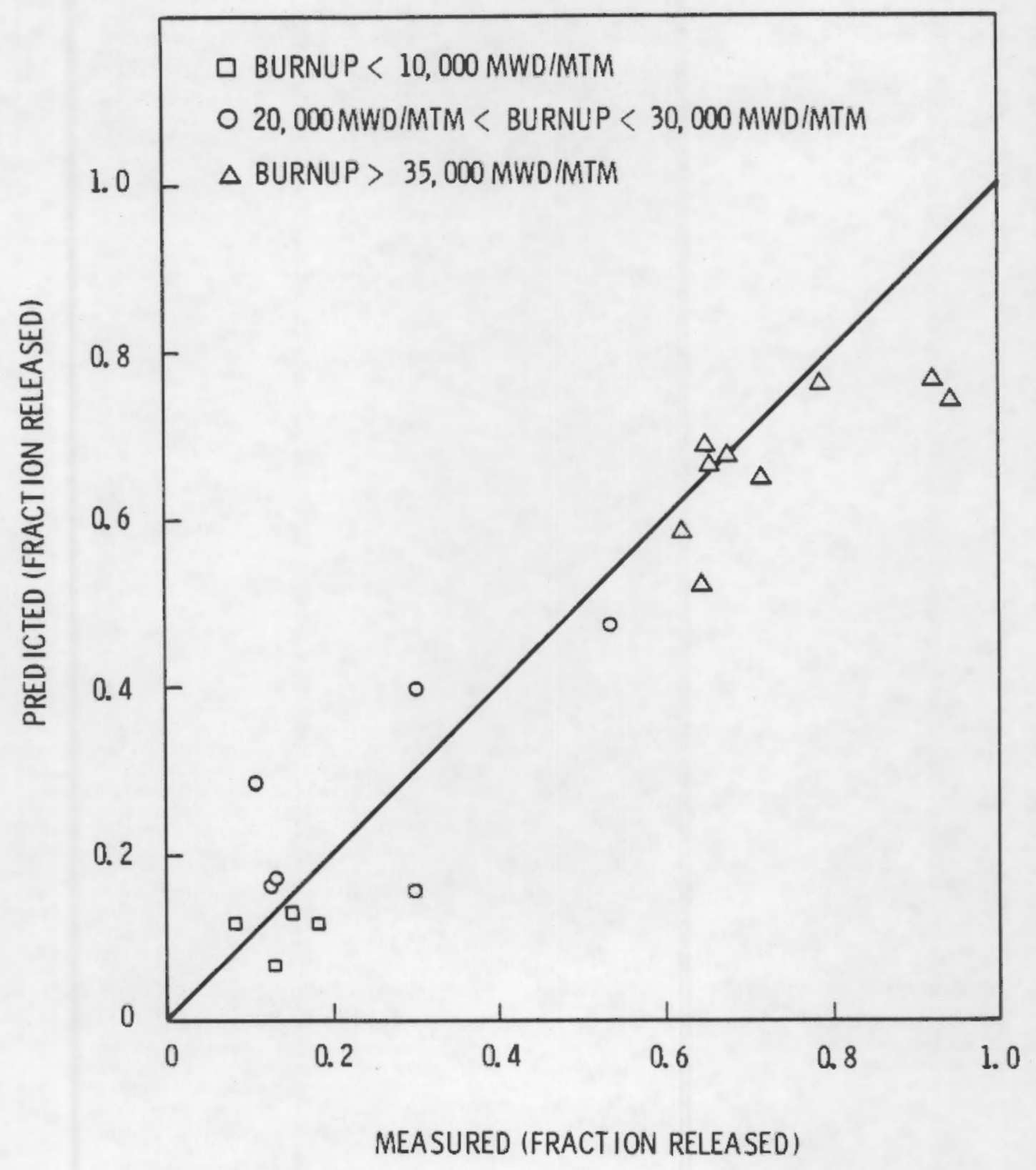

FIGURE 2. Comparison of ANS-5.4 With EBR-II Data 
TABLE 1.

COMPARISON OF ANS-5.4 WITH LOW BURNUP DATA

\begin{tabular}{|c|c|c|c|c|c|c|c|c|}
\hline & & Burnup & Power & & rature & & $\%$ Gas & lease \\
\hline & Rod & MWd/MTM & $\mathrm{kw} / \mathrm{m}$ & Surface & $\mathrm{CL}$ & $C L^{*}$ & Measured & Predicted \\
\hline 1 & CYRANO-II & 1033 & 45.6 & 1031 & 2344 & 2399 & 15.0 & 17.0 \\
\hline 2 & CYRANO-VIII & 7409 & 38.0 & 1116 & 2243 & 2296 & 13.0 & 19.7 \\
\hline 3 & $116-5$ & 4579 & 62.0 & 782 & 2580 & 2635 & 27.5 & 30.8 \\
\hline 4 & $117-1$ & 8713 & 64.9 & 822 & 2553 & 2613 & 25.4 & 36.0 \\
\hline 5 & ELP-4 & 11348 & 40.3 & 870 & 2046 & 2042 & 10.8 & 16.2 \\
\hline 6 & ELP-5 & 3870 & 44.3 & 763 & 1992 & 1985 & 4.9 & 4.7 \\
\hline 7 & ELP-6 & 13913 & 49.5 & 935 & 2462 & 2465 & 23.7 & 37.1 \\
\hline 8 & ELP-9 & 18804 & 48.9 & 859 & 2325 & 2319 & 25.8 & 34.0 \\
\hline 9 & ELP-10 & 9348 & 48.9 & 879 & 2357 & 2353 & $20: 3$ & 26.5 \\
\hline 10 & ELP-12 & 8424 & 43.6 & 934 & 2264 & 2262 & 19.7 & 23.9 \\
\hline 11 & LFL & 2230 & 51.2 & 728 & 1935 & 2003 & 5.7 & 7.5 \\
\hline 12 & LFF & 2230 & 50.8 & 833 & 2175 & 2249 & 17.3 & 18.4 \\
\hline 13 & LFB & 2230 & 49.5 & 915 & 2321 & 2405 & 23.4 & 26.6 \\
\hline 14 & LFS & 3120 & 71.8 & 834 & 2726 & 2819 & 37.9 & 37.5 \\
\hline 15 & LFW & 3290 & 75.8 & 705 & 2615 & 2712 & 24.8 & 31.1 \\
\hline 16 & LFT & 3290 & 72.8 & 827 & 2884 & 2983 & 49.6 & 41.5 \\
\hline 17 & LFX & 3290 & 75.4 & 792 & 2826 & 2927 & 36.8 & 39.2 \\
\hline 18 & LFM & 3030 & 69.9 & 714 & 2465 & 2558 & 15.5 & 27.1 \\
\hline 19 & LFH & 3030 & 69.2 & 806 & 2678 & 2774 & 31.1 & 35.6 \\
\hline 20 & LFD & 3030 & 67.9 & 893 & 2846 & 2939 & 45.8 & 42.3 \\
\hline 27 & CBN & 2650 & 56.1 & 728 & 2095 & 2135 & 12.3 & 11.1 \\
\hline 22 & CBO & 2670 & 56.7 & 735 & 2130 & 2177 & 14.9 & 12.6 \\
\hline 23 & CBP & 2610 & 55.1 & 730 & 2087 & 2133 & 14.1 & 10.9 \\
\hline 24 & CBR & 2710 & 57.1 & 769 & 2224 & 2273 & 15.7 & 17.0 \\
\hline 25 & CBT & 2620 & 54.4 & 767 & 2163 & 2213 & 15.3 & 14.4 \\
\hline 26 & CBV & 2760 & 50.8 & 741 & 2216 & 2265 & 16.5 & 16.2 \\
\hline 27 & CBY & 2630 & 54.4 & 790 & 2232 & 2289 & 16.9 & 17.9 \\
\hline 28 & CBX & 2710 & 56.1 & 796 & 2295 & 2347 & 18.8 & 20.4 \\
\hline 29 & DFE & 794 & 114.8 & 689 & 3499 & 3596 & 38.8 & 42.9 \\
\hline 30 & OFH & 648 & 94.8 & 712 & 3114 & 3209 & 31.1 & 34.9 \\
\hline 31 & DFD & 658 & 95.4 & 747 & 3180 & 3274 & 31.7 & 37.3 \\
\hline 32 & DFB & 528 & 78.4 & 735 & 2742 & 2834 & 16.9 & 25.3 \\
\hline 33 & DFA & 386 & 57.1 & 675 & 2003 & 2078 & 4.6 & 3.5 \\
\hline 34 & $4110-A E 1$ & 7051 & 59.4 & 887 & 2571 & 2636 & 21.6 & 37.3 \\
\hline 35 & 4110-AE2 & 6860 & 57.7 & 843 & 2450 & 2504 & 22.1 & 32.7 \\
\hline 36 & $4110-B E 7$ & 5738 & 49.5 & 821 & 2751 & 2199 & 13.9 & 19.7 \\
\hline 37 & $4110-B E 2$ & 7215 & 58.4 & 759 & 2322 & 2377 & 15.9 & 26.0 \\
\hline 38 & $4112-A E 1$ & 3795 & 64.0 & 692 & 2401 & 2458 & 12.6 & 22.1 \\
\hline 39 & 4112-AE2 & 3549 & 58.1 & 755 & 2333 & 2385 & 11.2 & 21.1 \\
\hline 40 & $4112-8 E 1$ & 3073 & 50.5 & 698 & 1974 & 2023 & 7.9 & 6.7 \\
\hline 41 & 4112-BE2 & 3313 & 54.4 & 735 & 2177 & 2230 & 12.6 & 14.4 \\
\hline 42 & $4113-A E 1$ & 3418 & 56.1 & 1027 & 2698 & 2755 & 26.7 & 40.0 \\
\hline 43 & 4113 -AE2 & 3116 & 51.2 & 1001 & 2551 & 2567 & 28.0 & 33.0 \\
\hline 44 & 4113-BE] & 3124 & 52.5 & 804 & 2240 & 2299 & 17.0 & 17.9 \\
\hline 45 & $4113-B E 2$ & 3181 & 52.2 & 956 & 2479 & 2533 & 21.0 & 30.7 \\
\hline
\end{tabular}

*Center-line temperature predicted by code 
TABLE 2.

COMPARISON OF ANS-5.4 WITH EBR-II OATA

\begin{tabular}{|c|c|c|c|c|c|c|c|}
\hline \multirow[b]{2}{*}{ Pin } & \multirow{2}{*}{$\begin{array}{l}\text { Burnup } \\
\text { MWd/MTM }\end{array}$} & \multirow{2}{*}{$\begin{array}{l}\text { Power } \\
\mathrm{kW} / \mathrm{m}\end{array}$} & \multicolumn{2}{|c|}{ Temperature ${ }^{\circ} \mathrm{K}$} & \multirow{2}{*}{$\frac{\cdot}{\underline{C L^{*}}}$} & \multicolumn{2}{|c|}{ \% Gas Release } \\
\hline & & & Surface & $\underline{\mathrm{CL}}$ & & Measured & Predicted \\
\hline 1 PNL $1-6$ & 8070 & 26.8 & 1042 & 1976 & 1866 & 12.9 & 6.8 \\
\hline 2 PNL 1-14 & 8180 & 27.7 & 1035 & 1996 & 2001 & 8.2 & 11.5 \\
\hline 3 PNL $1-17$ & 8680 & 29.4 & 1042 & 2056 & 2019 & 15.1 & 12.8 \\
\hline 4 PNL 1-18 & 8950 & 30.3 & 1039 & 2060 & 1990 & 18.1 & 11.7 \\
\hline 5 PNL $1-19$ & 9090 & 30.3 & 1128 & 2111 & 2030 & 29.9 & 15.7 \\
\hline 6 PNL $3-8$ & 26340 & 15.9 & 1198 & 1748 & 1723 & 10.7 & 28.3 \\
\hline 7 PNL 3-27 & 26740 & 15.4 & 1096 & 1626 & 1638 & 12.5 & 16.1 \\
\hline 8 PNL 3-33 & 26710 & 15.6 & 1124 & 1659 & 1635 & 13.1 & 16.9 \\
\hline 9 PNL 4-1 & 42140 & 28.0 & 1124 & 2024 & 2043 & 62.2 & 58.1 \\
\hline 10 PNL 4-26 & 40980 & 26.4 & 1119 & 2040 & 1913 & 64.5 & 52.1 \\
\hline 11 PNL 4-34 & 39570 & 25.4 & 1240 & 2124 & 2082 & 65.4 & .66 .5 \\
\hline 12 PIIL 7-10 & 24250 & 27.7 & 1135 & 1995 & 2030 & 30.1 & 38.9 \\
\hline 13 PNL 8-11 & 58200 & 39.9 & 1143 & 2351 . & 2375 & 92.2 & 76.5 \\
\hline 14 PNL 8-25 & 57180 & 38.1 & 1126 & 2272 & 2298 & 94.3 & 73.9 \\
\hline 15 PNL 8-37 & 55720 & 35.3 & 1091 & 2143 & 2184 & 65.2 & 68.7 \\
\hline 16 PNL $8-38$ & 27760 & 37.6 & 1070 & 2194 & 2275 & 53.6 & 47.3 \\
\hline 17 PNL 10-15 & 60550 & 28.6 & 1139 & 2061 & 2184 & 78.7 & 76.1 \\
\hline 18 PNL $10-23$ & 49820 & 27.4 & 1150 & 2090 & 2099 & 67.5 & 67.5 \\
\hline 19 PNL 10-63 & 51960 & 27.3 & 1120 & 2070 & 2010 & 71.5 & 64.9 \\
\hline
\end{tabular}

*Center-line temperature predicted by code. 
TABLE 3. Listing of the ANS54 Subcode.

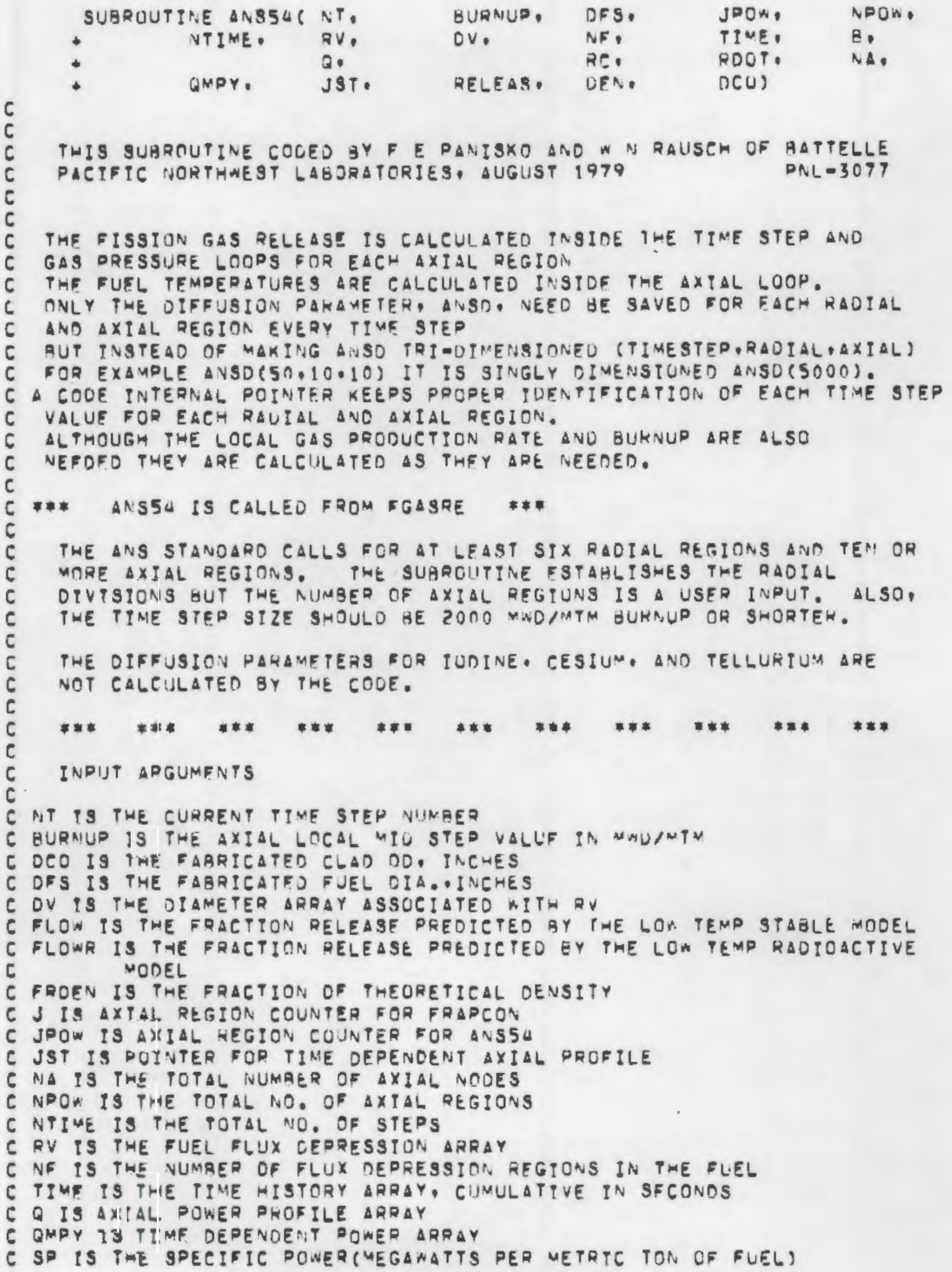




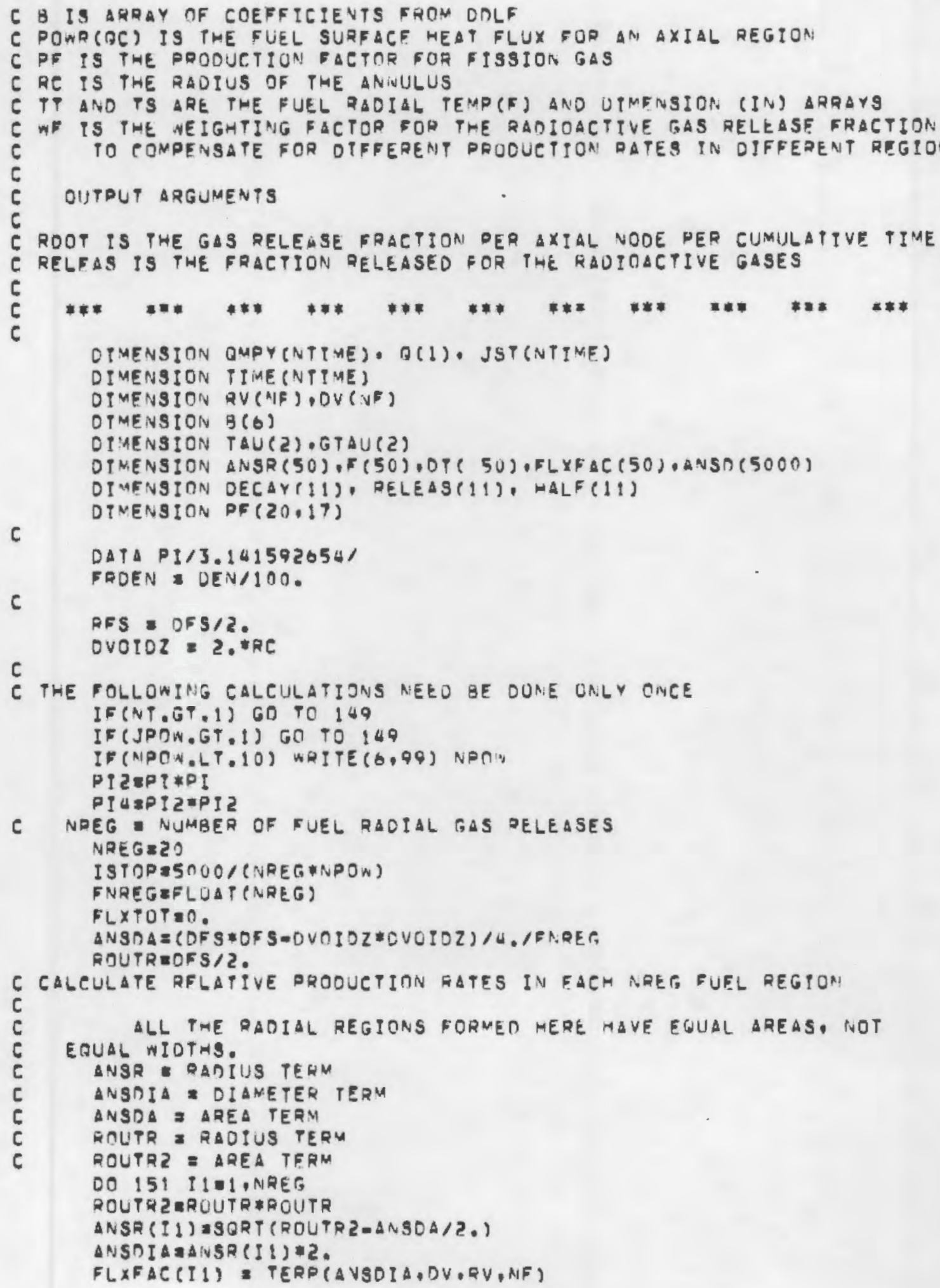




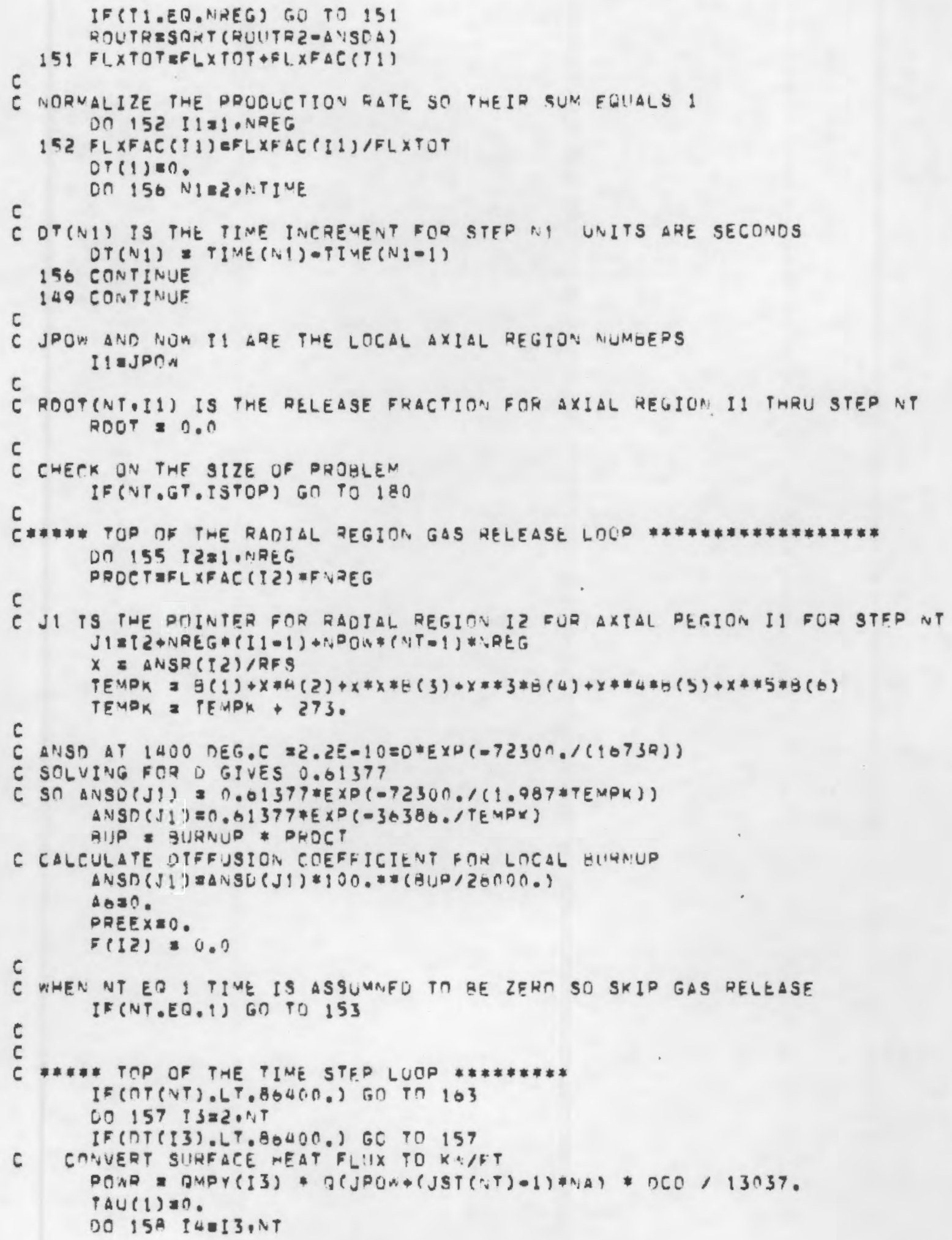




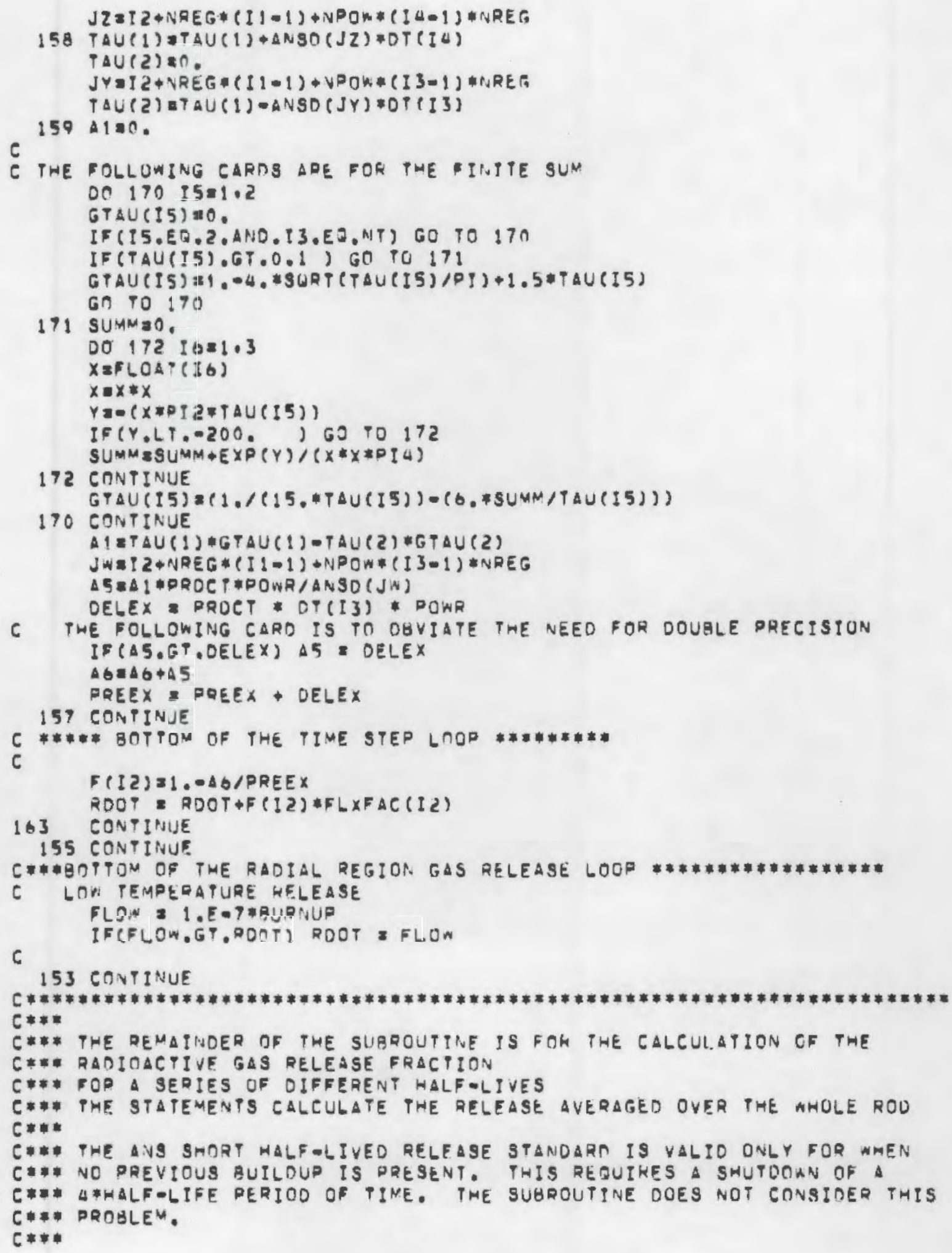




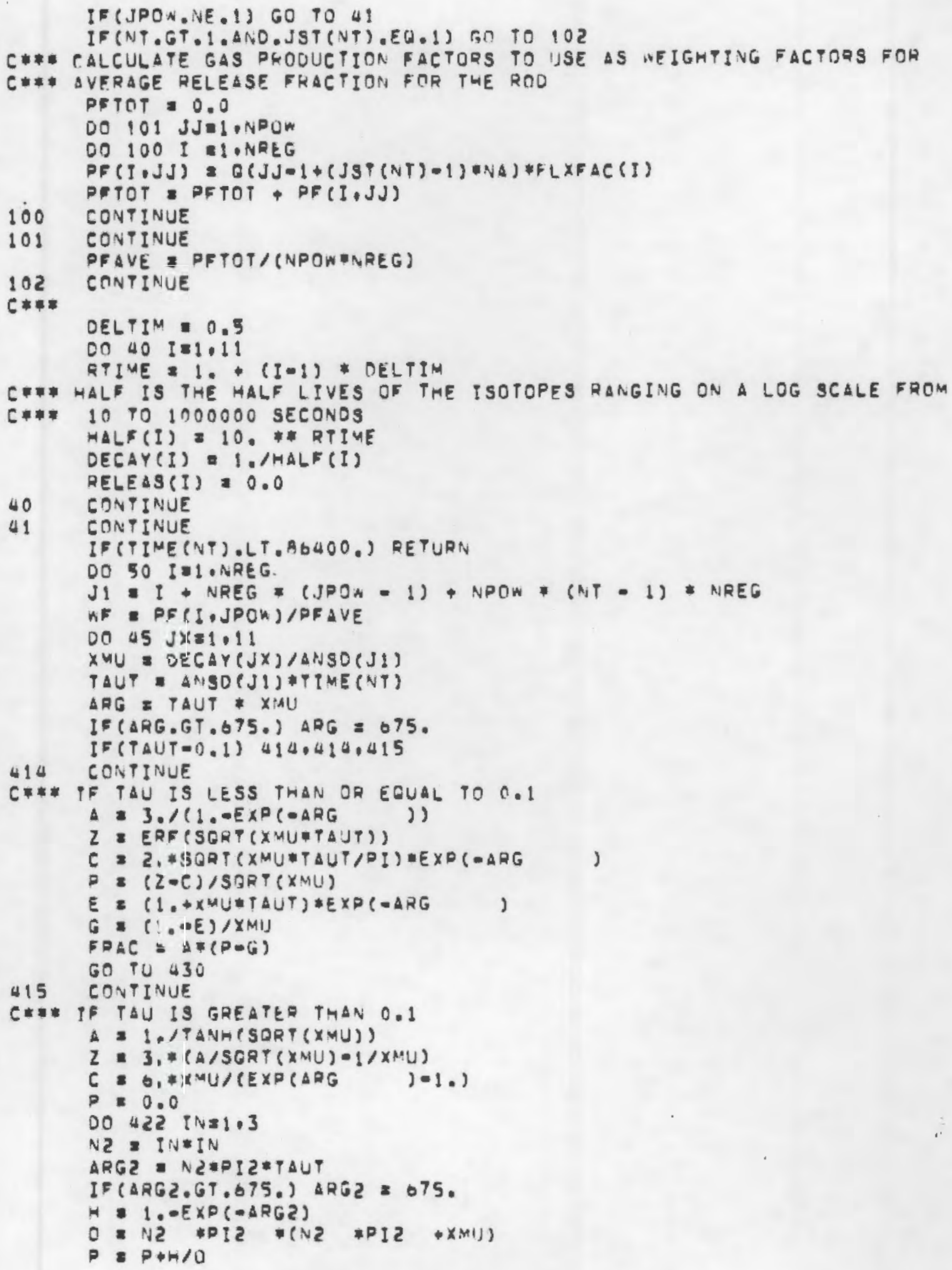




\section{TABLE 4. Listing of Subroutine GASPLT}

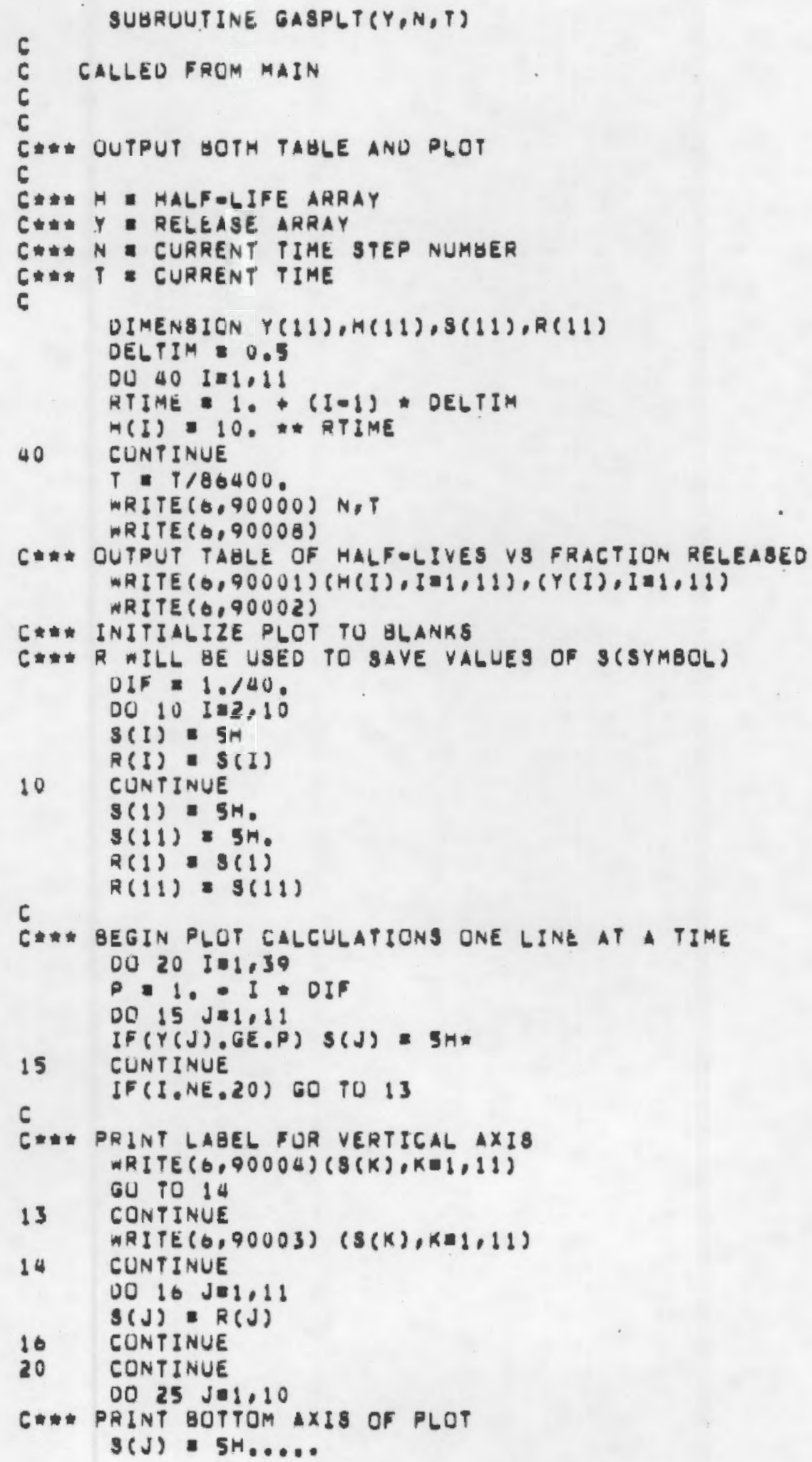




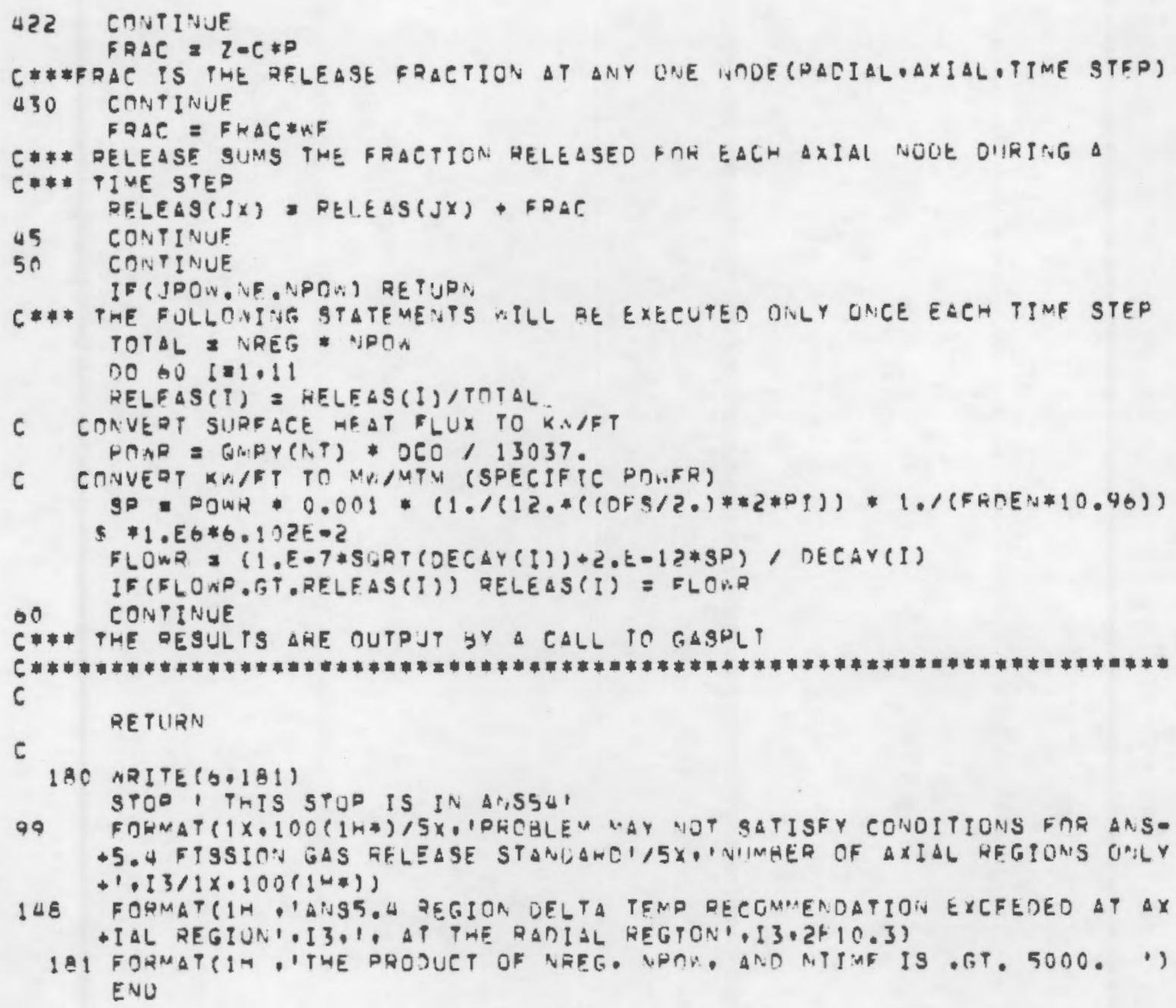




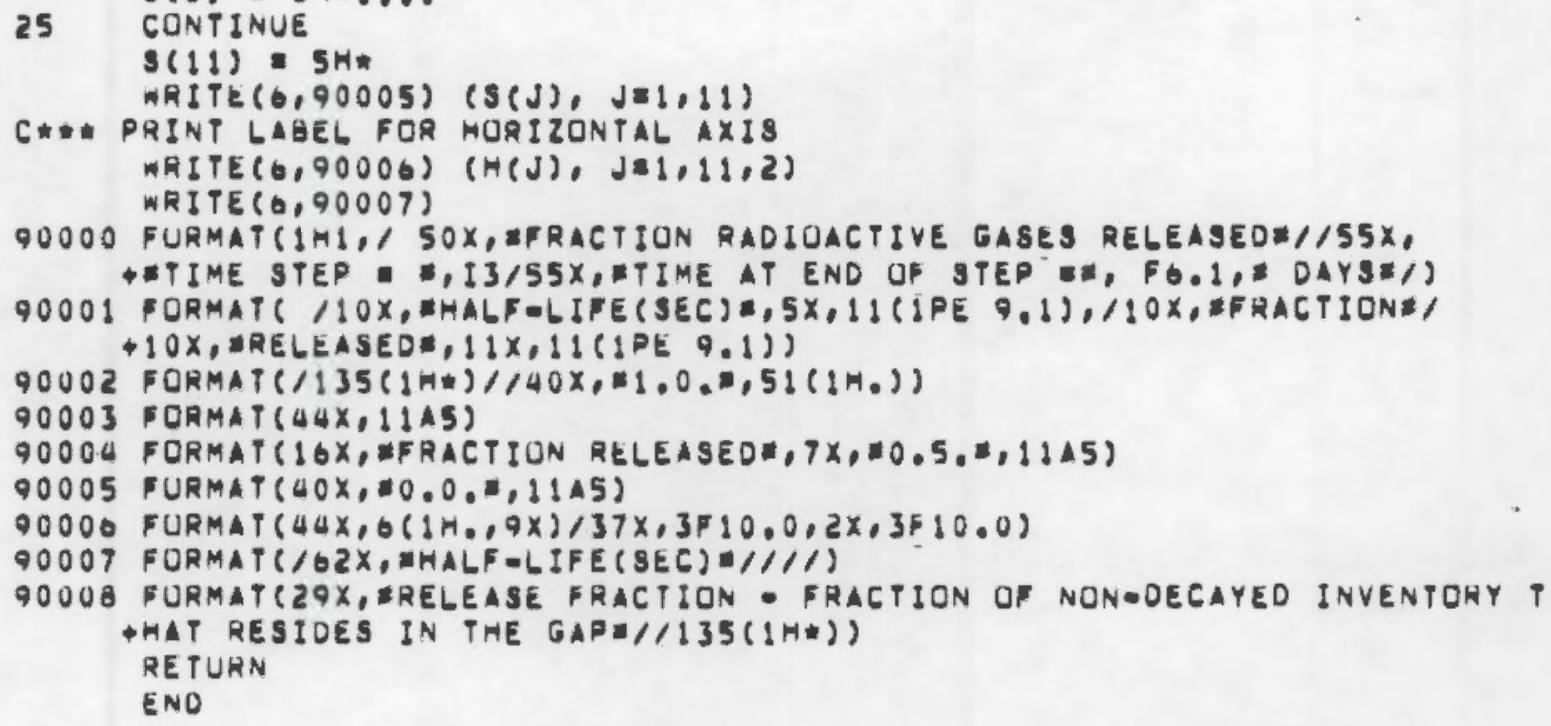


No. of

Copies

OFFSITE
No. of

Copies

OFFSITE
Karl Kniel

Chief, Core Performance Branch

Division of Systems Safety

U.S. Nuclear Regulatory

Commission

Washington, D.C. 20555

10
J. C. Voglewede
Reactor Fuel Section
Core Performance Branch
Division of Systems Safety
U.S. Nuclear Regulatory Commission
Washington, D.C. 20555
G. P. Marino
Fuel Behavior Research Branch
Division of Reactor Safety Research
Office of Nuclear Regulatory Research
U.S. Nuclear Regulatory. Commission
Washington, D.C. 20555

M. P. Bohn

EG\&G

550 2nd St.

Idaho Falls, ID 83401

W. F. Domenico

EG\&G

550 2nd St.

Idaho Falls, ID 83401

R. 0. Meyer

Core Performance Branch

Division of Systems Safety

U.S. Nuclear Regulatory

Commission

Washington, D.C. 20555
G. A. Berna

EG\&G

550 2nd St.

Idaho Falls, ID 83401

Or. M. Tokar

Reactor Fuels Section

Core Performance Branch

Department of Licensing

U.S. Nuclear Regulatory Commission

Washington, D.C. 20555

A. A. Churm

DOE Patent Oivision

9800 S. Cass Ave.

Argonne, IL 60439

30

U.S. Nuclear Regulatory Commission

Division of Technical Information and Document Control 7920 Norfolk Avenue

Bethesda, MD 20014
ONSITE

20
Pacific Northwest Laboratory

E. R. Bradley

M. E. Cunningham

C. R. Hann

D. D. Lanning

C. L. Mohr (5)

C. Nealley

F. E. Panisko

W. N. Rausch

R. E. Williford

Tech. Info. Files (5)

Publishing Coordination (2) 